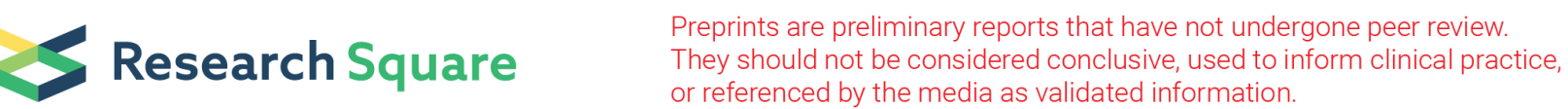

\section{Zhengyuan capsule for the treatment of cancer- related fatigue in lung cancer patients undergoing operation: study protocol for a randomized controlled trial}

\section{Zhouji Zhang}

Shanghai Chest Hospital; Shanghai Chest Hospital Shanghai JiaoTong University

Ming Zhang

Shanghai Chest Hospital; Shanghai Chest Hospital Shanghai JiaoTong University

Xiaoting Wu

Shanghai Chest Hospital; Shanghai Chest Hospital Shanghai JiaoTong University

Qing Cui

Shanghai Chest Hospital; Shanghai Chest Hospital Shanghai JiaoTong University

Yijun Guo (D 18017320230@163.com )

\section{Study protocol}

Keywords: Traditional Chinese medicine, Cancer-related fatigue, Postoperative lung cancer, Zhengyuan capsule, Randomized controlled trial

Posted Date: August 9th, 2019

DOI: https://doi.org/10.21203/rs.2.12517/v1

License: (c) (i) This work is licensed under a Creative Commons Attribution 4.0 International License.

Read Full License 


\section{Abstract}

Background: Cancer-related fatigue (CRF) is one of the most common and painful symptoms in patients with lung cancer undergoing treatment with operation, affecting patients' physical, psychological, family and social functions as well as their quality of life. However, there is very little evidence for treatment guidelines at home and abroad. Traditional Chinese Medicine (TCM) has been proved to improve the symptoms of general weakness and shown to be beneficial to cancer patients' recovery. TCM treatment should be based on syndrome differentiation. Aiming at the CRF, we should focus on bolstering the deficiency while simultaneously attacking and reinforcing it. This trial preliminarily evaluated the efficacy and safety of Zhengyuan capsule for CRF in lung cancer patients undergoing operation. Methods/design: The single center clinical study is a two-group, double-blind, prospective, placebo-controlled, randomized controlled trial (RCT) designed to assess the efficacy and safety of Zhengyuan capsule for CRF in lung cancer patients undergoing operation. Eligible participants will randomly divided into two groups: a treatment group receiving a 8-week Zhengyuan capsule regimen therapy and a control group receiving a 8-week placebo capsule regimen therapy. The main efficacy indicator is fatigue, assessed using severity scores from the Cancer Fatigue Scale(CFS) measurement system. Secondary efficacy measures include fatigue which assessed using severity scores from the Multidimensional Fatigue Symptom InventoryShort Form(MFSI-SF) measurement system, TCM syndrome pattern differentiation, levels of immunologic indicators(TNF-a, IL-6, IL-1, T lymphocytes subsets and B lymphocyte subsets), patient's pulmonary functionality and adverse events (AEs). Evaluation will be carried out at the screening period, baseline, the middle of the intervention and the end of the intervention, respectively. Discussion: Experimental results of Zhengyuan capsule can guide the improvement of CRF and provide evidences that Zhengyuan capsule is effective and safe in lung cancer patients undergoing operation. The data can be imported into the management and treatment guidelines of CRF for patients with lung cancer undergoing operation throughout China nationwide. Trial registration: Chinese Clinical Trial Registry (chictr.org.cn), ChiCTR1900022712/YZJ-TCM-ZYJN-02. Registered on 23 April 2019.

\section{Background}

Lung cancer is one of the most common malignancies with highest morbidity and mortality worldwide ${ }^{[1]}$. According to histopathologic classification, it is divided into two categories (non-small-cell lung cancer and small-cell lung cancer). Surgical operation is an important pillar of Multi-Disciplinary Treatment (MDT) for non-small-cell lung cancer (NSCLC). The basic principle of tumor surgery is to reveal farthest excision of pulmonary tumor and farthest preservation of organ function. Surgical treatment is traumatic stress, which leads to increased sympathetic nervous activity and adrenaline level. Patients' mental and psychological is in a state of nervous and anxiety for a long time, which causes fatigue. These fatigue symptoms are different from normal fatigue, which have characteristics about rapid and serious occurring, long time keeping, huge energy wasting, unpredictable events and cannot be relieved by rest. It is defined as CRF, which is not the body's protective compensation reaction. 
Patients with lung cancer undergoing treatment with operation have CRF, which have a series of subjective sensations about lack of energy, weakness, lethargy, lethargy, fatigue, lack of activity, intolerance, lack of concentration, lack of motivation, lack of interest, loss of memory, depression, inability to do the work, etc. CRF is one of the predictors of cancer survival[2]. CRF seriously affects patients' physical, psychological, family and social functions as well as their quality of life $\mathrm{f}^{[3]}$.

Because of the frequency of CRF in lung cancer patients undergoing operation, non-drug and drug interventions for CRF are widely verified in experiments and used in clinical. Non-drug interventions include health educations, sports and psychological behaviors. Drug interventions include central nervous system (CNS) stimulant, cortiso, erythropoietin, antidepressant and TCM. TCM focus on multiple treatments in CRF condition, such as herbal medicine, acupuncture, moxibustion, etc. Herbal medicine, as a preventive agent or adjuvant treatment, has been shown to significantly reduce the incidence of CRF. Other mechanisms by herbal medicine include retarding cancer progression, boosting the immune system, and ameliorating operation-induced complications and side effects, such as pain ${ }^{[4-5]}$.

Theory of TCM has been widely used in the treatment of lung cancer patients undergoing operation in China. Ancient sages formulate therapeutic compendium depletion of essence causing deficiency and treatment focus on spleen and kidney. Lung cancer patients not only have manifestation of deficiency because of tumor invasion or treatment, but also the basement on excess because of tumor itself. So, the treatment plan is based on replenishing qi to invigorate the spleen and reinforcing kidney to replenish essence, and supplemented by both regulating qi-flowing for eliminating phlegm and resolving hard lump. Modern physicians take CRF as 'consumptive disease' to treat, which is mainly caused by deficiency of qi and disharmony of yin and yang.

One hypothesis proposes that cancer disrupts the normal Hypothalamic-pituitary-adrenal(HPA) axis either directly or indirectly causing endocrine changes that induce CRF. The changes in the HPA axis in cancer could be secondary to other various disease-related factors like surgery and excess proinflammatory cytokines (IL-1, IL-6, and TNF-a) ${ }^{[6]}$.

Dysregulated inflammation and its toxic downstream effects are considered to constitute significant biological bases for CRF and cancer-related symptoms(such as pain, loss of appetite, lethargy, insomnia and depression.etc. $)^{[7-8]}$. Cancer treatment may lead to important long-term changes in the cellular immune system ${ }^{[9]}$. Preclinical research on immune-to-brain communication pathways in the peripheral immune system indicates that proinflammatory cytokines, primarily interleukin(IL) $-1 \beta$ and tumor necrosis factor (TNF)-a send signals to the brain and then give rise to pathological behaviours (including fatigue, sleep disturbances and depressive symptoms ${ }^{[10]}$. Animal experimental studies that elevated levels of various markers for inflammation(IL-6, IL-10 and TNF- $\mathrm{a}$ ) increase weariness and decreased motor activity ${ }^{[11]}$.

Zhengyuan capsule was developed by Yangtze River Pharmaceutical Industry co. Ltd. The character of Zhengyuan capsule is warm but not dry, enriching but not slimy, which aim at the etiology and 
pathogenesis of CRF. The compositions of Zhengyuan capsule are epimedium, dried raw ginseng, honeyfried astragalus, atractylodes macrocephala, tortoise shell powder, turtle shell powder, wine-processed glossy privet fruit and dried orange peel. Epimedium, which was the monarch herb, promotes proliferation of $\mathrm{T}$ lymphocytes and secretion of cytokines(IL-2 and TNF) ${ }^{[12-13]}$. The minister herbs include dried raw ginseng, honey-fried astragalus and atractylodes macrocephala. Dried raw ginseng contains ginsenosides, which can alleviate fatigue. An abstract presented at the 2003 meeting of the American Society of Clinical Oncology reported that ginseng significantly improved total and average fatigue levels in people with cancer ${ }^{[14]}$. Astragalus increases the content of cortisol in plasma and has a significant regulatory effect on adrenal glands ${ }^{[15]}$. Atractylodes macrocephala increase $\mathrm{T}$ lymphocyte numbers and promote secretion of cytokines(IL-2 and TNF $)^{[16-17]}$. The adjuvant herbs include tortoise shell powder, turtle shell powder and wine-processed glossy privet fruit. Glossy privet fruit proliferates mouse spleen cell and raises the level of IL-2. TCM believed that tortoise shell and turtle shell are the love of flesh and blood products, which are the major tonic for consumptive disease.

This study aims to assess the efficacy and safety of the addition of Zhengyuan capsule for lung cancer patients undergoing operation. The primary hypothesis is that the Zhengyuan capsule (here our treatment group) will experience less fatigue, as measured using severity scores from the CFS measurement system, than the placebo capsule (here our control group). Secondary objectives are to evaluate the efficacy of Zhengyuan capsule on the following factors: fatigue which assessed using severity scores from the MFSI-SF measurement system, TCM syndrome pattern differentiation, levels of immunologic indicators(TNF-a, IL-6, IL-1, T lymphocytes subsets and B lymphocyte subsets), patient's pulmonary functionality and AEs.

\section{Methods/design}

\section{Design}

This study is registered on chictr.org.cn (ChiCTR1900022712). A program summary of the whole experiment is shown in Fig.1, and the test flow chart is provided in Fig.2. The single center clinical study is a two-group, double-blind, prospective, placebo-controlled, RCT to evaluate the efficacy and safety of Zhengyuan capsule for CRF in lung cancer patients undergoing operation. Eligible participants will be randomized to either a treatment group receiving a 8-week Zhengyuan capsule regimen or a control group receiving a 8-week placebo capsule regimen. The protocol include clinical research directory and Additional files (the CFS checklist, the MFSI-SF checklist and the evaluation scale of TCM qi syndrome pattern).

\section{Setting and participants}

The study will be conducted in Shanghai. Patients will be recruited from the department of integrated Chinese and western medicine in Shanghai Chest Hospital. Patients who meet all inclusion criteria are 


\section{Randomization, allocation concealment, and blinding}

After screening, patients should sign and provide informed consent. They will be randomized into either a treatment group receiving a 8-week Zhengyuan capsule regimen or a control group receiving a 8-week placebo capsule regimen. A block randomization sequence will be generated by SAS 9.4(SAS Institute Inc., Cary, NC, USA) in a 1:1 ratio.

The clinician and relevant medical staff obtained the random number set by statistics and filled in 'the random number' of the study cases. Then the drug distributor applied for a trial drug based based on a random number. The web-based allocation system would display the drug number to be issued. Because of giving simulated and real capsules having the same size, color and smell, it is possible to blind the patients and clinicians and relevant medical staffs. Project sponsor, experimental technicians, outcome analysts, data managers and statisticians will be unaware of the treatment allocations.

\section{Diagnostic criteria for CRF}

Refer to the diagnostic criteria of CRF in the tenth conference for the revision of International Classification of Diseases (ICD-10), fatigue symptoms recur for more than two weeks and concomitant with five or more of the following symptoms: 1.weakness or heaviness in the body; 2.attention can't concentrate; 3.lack of enthusiasm, depression and decreased interest; 4.insomnia or lethargy; 5 .both sleep and rest are fine, but don't feel refreshed; 6 .difficulty in movement; 7 . There are emotional responses, such as sadness and frustration; 8.inability to perform routine activities that were previously adequate; 9 .short-term memory loss; 10 .fatigue symptoms persist for hours without relief

\section{Eligibility criteria}

\section{Inclusion criteria}

Participants who meet the following criteria are eligible:

1. Histologically or cytologically confirmed lung cancer; Clinical confirmed cancer related fatigue, and Chinese version of Cancer Fatigue Scale(CFS) score of $\geq 40$ and $₫ 60$;

2. Age $\otimes 18$ and $\leq 75$ years, male or female; 
3. With Stage I lung cancer, status-post surgery with R0-resection between 3 weeks and 1 month, and chemoradiotherapy is not required;

4. The Eastern Cooperative Oncology Group (ECOG) performance status (PS) score of $\leq 2$ points;

5. Life expectancy $\geq 6$ months;

6. Informed consent form signed voluntarily.

\section{Exclusion criteria}

Exclusion criteria are as follows:

1. Pregnant, breastfeeding or plan to pregnant;

2. Allergic constitution or allergic to the ingredients of this preparation;

3. Cognitive impairments or verbal expression defects, which are difficult to cooperate with the investigator;

4. Suspected or confirmed drug abuse or alcoholism;

5. Combined severe cardiovascular, digestive, renal, endocrine, hematopoietic, neurological or chronic disease, which may affect effect judgment;

6. Abnormal liver function (ALT or AST $\geq 2$ times Upper limits of normal); abnormal renal function (serum creatinine level is above upper limits of normal);

7. Participated in other clinical trials within three months;

8. The investigators considered that inappropriate for inclusion

\section{Combination therapy}

\section{Banned drugs}

1. Erythropoietin(EPO), methyl ester, selective serotonin reuptake inhibitor(Ssri), amantadine(AMA), glucocorticoid, melatonin and octreotide.

2. Immunotherapy, including mycobacterium vaccine, staph vaccine, sizofiran and cytokine antagonists.

3. Except for experimental drugs, it is forbidden to use TCM decoction or proprietary chinese medicine with therapeutic effect on CRF, including oral and external dosage forms.

\section{Combined medication requirements}


Person's underlying diseases and combined medications should be recorded in the chart. Keep detailed records of reasons for combination therapy, drug name, the dose and frequency of taking the medicine, total daily dose, start date, end date and medication at last visit.

\section{Interventions and control}

The treatment delivery processes for both the intervention and control groups are outlined in Fig. 2 .

\section{Control group}

Patients in the control group will receive a 8-week placebo capsule regimen. Placebo capsule, as Zhengyuan simulated capsule, will be manufactured by Guangzhou Hairun Pharmaceutical Co., Ltd., based on good manufacturing practice (GMP) standards. It will be administered at a dose of $1.8 \mathrm{~g} \mathrm{(4}$ capsules) by peros thrice daily for 8 weeks as a period.

\section{Intervention group}

Patients in the treatment group will receive a 8-week Zhengyuan capsule regimen. Zhengyuan capsule will be manufactured by Guangzhou Hairun Pharmaceutical Co., Ltd., based on good manufacturing practice (GMP) standards. Each capsule contains epimedium, dried raw ginseng, honey-fried astragalus, atractylodes macrocephala, tortoise shell powder, turtle shell powder, wine-processed glossy privet fruit and dried orange peel. It will be administered at a dose of $1.8 \mathrm{~g}$ ( 4 capsules) by peros thrice daily for 8 weeks as a period.

\section{Data collection}

The study data collection process is outlined in Fig.2. The investigator participating in the clinical trial must be qualified, and have the professional background and ability to conduct the clinical trial.

\section{Patient background and medical history}

the screening period, that three weeks to one month after the surgery: (1)demographic data; (2)combination therapy and medical history; (3)specified physiological tests; (4)laboratory tests

\section{Outcome measures and assessments}


Each participant will be asked to attend an in-person assessment appointment at the department of integrated Chinese and western medicine in Shanghai Chest Hospital at four time points: day-7 0(the screening period), day 0 (baseline), day 28 \pm 2(the middle of the intervention) and day 56 \pm 2 (the end of the intervention). During each evaluation, participants will be asked to complete specified physiological tests, self-reported questionnaires, laboratory examinations or tests. Patients will fill in self-reported questionnaires under the guidance of clinicians or relevant medical staffs. And all reports will be administered by outcome analysts.

\section{Primary outcome}

\section{Fatigue severity on CFS Chinese version grading}

Fatigue severity will be self-assessed using the CFS Chinese version at the screening period, baseline, the middle of the intervention and the end of the intervention. CFS has good validity and reliability. It is easy to perform and should be a good instrument for the evaluation of the cancer-related fatigue in the Chinese cancer patients. The total score for CFS is the sum of three domains (physical, emotional and cognitive). Its related symptoms on a scale of 1 to 5 (1, no; 5 , very much).

\section{Secondary outcomes}

Secondary outcomes include the total score for MFSI-SF Chinese version grading, TCM syndrome pattern differentiation, levels of immunologic indicators(TNF-a, IL-6, IL-1, T lymphocytes subsets and B lymphocyte subsets), patient's pulmonary functionality and adverse events (AEs). The secondary outcomes are listed below.

\section{Fatigue severity on MFSI-SF Chinese version grading}

Fatigue severity will be self-assessed using the MFSI-SF Chinese version at the screening period, baseline, the middle of the intervention and the end of the intervention. MFSI-SF has good validity and reliability. It is easy to perform and should be a good instrument for the evaluation of the cancer-related fatigue in the Chinese cancer patients. The total score for MFSI-SF is the sum of the four domains (conventional, physical, emotional and mental) minus the value of the energic domain. MFSI-SF is a six-item subscale to measure the subjective experience of fatigue. Answers are on a 5-point scale, ranging from 0, 'not at all', to 4 , 'extremely'. 


\section{TCM syndrome pattern differentiation}

TCM believes that CRF belongs to the category of 'consumptive disease'. Ancient sages and saints consider the depletion of essence causing deficiency as principle of treatment. The diagnosis as described in TCM, deficiency of qi syndrome pattern, will be based on guidelines delineated in the Clinical Research of New Investigational Drugs in Traditional Chinese Medicine ${ }^{[18]}$. The total score for TCM syndrome pattern differentiation is the sum of two domains (primary and secondary signs and symptoms). Primary signs and symptoms including fatigue, listlessness and shortness of breath. Secondary signs and symptoms including laziness to speak and spontaneous perspiration. The evaluation scale of deficiency of qi syndrome pattern is on a 4-point scale, ranging from 0 , 'not at all', to 3 , 'severity'.

\section{Levels of immunologic indicators}

Blood samples will be collected to measure the levels of immunologic indicators. TNF-a, IL-6, IL-1, T lymphocytes subsets, B lymphocyte subsets and NK cell subsets will be analyzed at the department of integrated Chinese and western medicine in Shanghai Chest Hospital. These are associated with cancerrelated fatigue in lung cancer patients undergoing operation.

\section{Pulmonary function(PE)}

PE include lung volume and pulmonary ventilation function. Lung cancer operation involves the removal of part or all of the lung. As a result, both lung volume and pulmonary ventilation function are significantly reduced. The patient may feel flustered and breathe hard after the operation. So they reduce the frequency of aerobic exercises, which has a intimate relationship with CRF.

\section{Adverse events (AEs)}

During this research, Data Safety Monitoring Committee will assess the progress and security data of this experiment. In order to assess security, we will perform the following tests on the baseline and the end of the intervention: vital signs, blood routine, urine routine, microscopic examination of urinary sediment, liver function, kidney function and electrocardiogram.

In particular, we will pay attention to adverse drug reactions (ADRs) and AEs associated with Zhengyuan capsule.

The prescription contains eight traditional Chinese medicines. Most of them belong to Chinese medicinal materials of tonic type, and part of them are combination of medicine and food. Single Chinese medicine has advantages of safe, effective and less toxic side effects, but some herbs have been listed as certain 
toxic and side effects in experimental research and clinical application, including jaundice, abnormal liver function, allergic diarrhea, skin rash, insomnia nervousness, high blood pressure, hypersexuality and hypofunction, headache, palpitations and bleeding.

AEs will be defined as any untoward medical occurrence that may present during treatment with a pharmaceutical product but which does not necessarily have a causal relationship with this treatment. Participants will be instructed to report AEs to the research scholar and the research scholar will monitor participants for potential occurrences of AEs. Patients should fill in the adverse event record form truthfully. AEs recorded include time of occurrence, severity, duration, effective measures taken and outcomes. All AEs will be followed up from the date they are brought to the researcher's attention until resolution. The severity of adverse events will be judged by National Cancer Institute Common Terminology Criteria for Adverse Events (NCI CTCAE, version 4.03.)

In case of serious adverse events(SAEs), researchers must report to the sponsor, medical ethics committee, local drug administration and national drug administration within 24 hours. SAEs will be defined according to the International Conference on Harmonization guidelines ${ }^{[19]}$ : any adverse event will be regarded as serious if it results in death, is life-threatening, requires hospitalization or prolongation of existing hospitalization, or results in persistent or significant disability or incapacity.

\section{Sample size estimation}

The primary endpoint is the fatigue severity self-assessed using the CFS Chinese version grading scales. According to the literature, compared with the baseline, the change of CFS was 14 points in the intervention group and 10 points in the control group. The standard deviation of both groups was 7 points. The test effectiveness $(1-\beta)$ was set at 0.8 , the inspection level a was set at 0.05 . The two-sample $t$ test process of PASS was used for calculation, then a sample size of 100 was required. Considering the $15 \%$ shedding rate, the final sample size has been calculate and expand at a total of 120 patients(60 in each group).

\section{Statistical analysis}

According to the intention-to-treat principle, the whole experimental analyses will be divided into enrolled cases, demographic date and baseline, medication compliance and combination, efficacy and safety. All statistical analyses will be performed using Statistical Analysis System software (SAS, version 9.4). All results made in this study are based on two-sided tests, and $a=0.05$. A P value $\leq 0.05$ will be considered statistically significant. The confidence levels for all confidence intervals are $95 \%$. Categorical data will be presented as percentages or frequencies and compared using the chi-square or Fisher's exact test, while Continuous data will be presented as means and standard deviations and compared using the independent t test or Wilcoxon's rank sum test. Data for subjects who meet the dropout criteria (i.e., 
incidence of serious AEs, $<80 \%$ or $120 \%$ compliance with the protocol, incomplete data that could influence the trial, reluctance to continue the study, large error in protocol, or deviation from the protocol) will be excluded. Missing values will be implemented by multiple imputations.

\section{Quality control and trial management}

The following must be done in this experimental research:

1. The investigator participating in the clinical trial must be qualified, and have the professional background and ability to conduct the clinical trial. The whole research team, including research investigators and assistants will be required to attend unified training and good command of research procedures. Researcher is fixed and do not change easily.

2. The original medical records collected must be complete and true.

3. All capsules are kept in the Good Clinical Practice (GCP) pharmacy, and a research assistant is responsible for distribution. To establish clinical trial drug use archives, including drug release date, Pinyin abbreviation of the subject's name, number of pills issued and recovered.

4. Investigators will enter data using the double-entry method. All observations are verified to ensure date reliability.

5. Laboratory quality control procedures and standard operating procedures(SOPs) will be checked regularly by research assistants.

6. Inspections of the whole experiment will be performed regularly by the Department of Integrated Chinese and Western Medicine of Shanghai Chest Hospital. The unit hold regular meetings on issues arising from work and feedback information in the form of reports.

7. Research investigators should improve patient compliance, conduct regular follow-up and control the subject withdrawal rate less than $20 \%$.

\section{Discussion}

CRF is closely related to the means of cancer treatment, which is a common and unavoidable side effect of current forms of cancer treatment. The basic principle of surgical treatment is to maximize the removal of tumor tissue and to maximize the preservation of organs and normal function of the body. Different surgical methods and surgical range bring about differences in CRF. Surgical treatment is traumatic stress, leading to increased sympathetic tone and adrenaline levels in the body. The above situation leads to the mental state of patients in a state of tension and anxiety, which causing CRF.

It interferes with daily activities of cancer patients and affects various aspects of life, including physiological, social, and psychological well being ${ }^{[20]}$. Non-drug and drug interventions for CRF are often used in clinical practice. Compared with the drug treatment of cancer-related fatigue in lung cancer 
patients undergoing operation (such as phenyl propanolamine side effect, cortisol, EPO and antidepressant, etc.) in the foreign country, TCM was mainly used to intervene CRF in china.

Both drugs and non-drugs can interfere with CRF, so this study has some limitations. Due to the nature of the non-drug intervention, health education, aerobic exercise or psychological behavior may be threaded through the whole process of the treatment, this will have some impact on the results of the experiment. We will make every effort to ensure that the use of EPO, methylphenidate, Ssri, AMA, glucocorticoid, Melatonin, Octreotide, immunotherapy and Chinese medicine with similar effects are prohibited.

Considering that Zhengyuan capsule is closely related to the characteristics of deficiency syndrome of TCM, which considered the constitution or specific characteristics of health status in prescribing medication, the concurrent use of outcome measurements reflecting the perspective of traditional medicine will be of interest in future studies.

Zhengyuan capsule is mainly used for those people with kidney qi deficiency in TCM, which is defined as a highly sensitive and highly fatigued condition ${ }^{[21]}$. Although fatigue is one of the typical symptoms of this qi-deficiency syndrome, other phenomenon of both deficiency and excess syndrome may also be considered in studying Zhengyuan capsule.

Though there are subtle holes in the subject, we still believe that this research will be positive and has the potential to contribute to the development of this proprietary Chinese medicine intervention to help relieve CRF. It will also establish feasibility and provide preliminary evidence on the efficacy and safety of Zhengyuan capsule for CRF in lung cancer patients undergoing operation. Based on the results of this subject, future studies should employ more subjects with different stages of surgery and include longterm administration (more than two weeks of medication) and follow-up (within five years).

\section{Trial status}

Recruitment started in May 2019, and it is expected to finish in May 2021. The protocol number of this clinical trial is YZJ-TCM-ZYJN-02. Version number is V1.1. Version date is 2019.04.23.

\section{Abbreviations}

ADRs: adverse drug reactions; AEs: adverse events; ALT: alanine aminotransferase; AMA: amantadine; AST: glutamic oxalacetic transaminase; CFS: Cancer Fatigue Scale; CRF: Cancer-related fatigue; CNS: central nervous system; ECOG: Eastern Cooperative Oncology Group; EPO: erythropoietin; GCP: Good Clinical Practice; GMP: good manufacturing practice; HPA: Hypothalamic-pituitary-adrenal; IL-1 $\beta$ : interleukin-1 $\beta$; IL-2: interleukin-2; MDT: Multi-Disciplinary Treatment; MD: mean difference; MFSI-SF: Multidimensional Fatigue Symptom Inventory-Short Form; NCI CTCAE: National Cancer Institute Common Terminology Criteria for Adverse Events; NSCLC: non-small-cell lung cancer; PE: Pulmonary function; RCT: randomized controlled trial; SAE: serious adverse event; Scr: serum creatinine; SD: standard deviation; 
SPIRIT: Standard Protocol Items: Recommendations for Interventional Trials; SOPs: standard operating procedures; SSRIs: selective serotonin reuptake inhibitors; TCM: Traditional Chinese Medicine; TNF-a: tumor necrosis factor-a;

\section{Declarations}

\section{Acknowledgements}

This work was jointly supported by Guangdong provincial department of finance(subject code: 2060502), the National Natural Science Foundation of China (81573893) and the Shanghai Municipal Commission of Health and Family Planning (2018LP026). We would like to express our sincere thanks to all the research investigators and assistants for their valuable support in participant recruitment.

\section{Funding}

This study is supported by the Yangtze River Pharmaceutical Industry co. Ltd. The funding agency coordinated the experiment and assist researchers of Shanghai Chest Hospital to complete the research steps, including designing research, collecting and analyzing date, writing the manuscript. People in this institution who are not involved in the project participated in the process of double-blind and RCT designing. At the same time, they left emergency letters for emergency unblinding.

\section{Authors' contributions}

All authors have made substantive contributions to this study in regard to design and implementation. $\mathrm{MZ}$ is responsible for conceiving and designing the trial. CQ is responsible for supervising and coordinating the clinical trial, making the final decision to terminate the trial. XW is responsible for participating recruitment and management. $Z Z$ is responsible for participating in data collection and drafting the manuscript. YG plays a key role in analyzing the data resulting and approving the final manuscript. All authors have read and approved the final manuscript.

\section{Ethics approval and consent to participate}

The trial protocol has been approved by the Research Ethical Committee of Shanghai Chest Hospital (approval no.LS1916). It will be monitored by the trial agency at Shanghai Chest Hospital. The project will be proceeded in accordance with the principles of the Declaration of Helsinki (2008 version). The study design will follow the principles set out in the GCP guidelines according to theories guiding the appropriate use of TCM in clinical applications. The research design accords with the Standard Protocol Items: Recommendations for Interventional Trials (SPIRIT) guidelines ${ }^{[22]}$ (see Additional file 1). Before 
entering the study, all participants should sign the informed consent, including the possible risks, procedures and knowledge of the purpose of the study.

The Ethics Committee of Shanghai Chest Hospital will review the study progress at least once a year. Any formal revision to the protocol required must be approved by the Ethics Committee of Shanghai Chest Hospital before implementation if modifications may affect the conduct of the study, potential benefit of the patient or patients' safety. For this RCT, trained research investigators or assistants will introduce and discuss the research information to eligible patients who wish to participate, and a signed informed consent form will be obtained before each participant's enrollment. Participants can quit at any time.

\section{Competing interests}

The authors declare that they have no competing interests.

\section{Author details}

Department of Integrated Chinese and Western Medicine, Shanghai Chest Hospital; Shanghai Chest Hospital Shanghai JiaoTong University, No. 241 Huaihai Road, Shanghai 200030, China

\section{Consent for publication}

Consent for publication refers to consent for the publication of identifying images or other personal or clinical details of participants that compromise anonymity. Seeing as this is not applicable to this manuscript, so I state "Not Applicable" in this section.

\section{Availability of data and material}

Seeing as this is not applicable to this manuscript, so I state "Not Applicable" in this section.

\section{References}

1. Siegel R, Naishadham D, Jemal A. Cancer statistics, 2013. CA Cancer J Clin.2013;63(1):11-30.

2. Gupta D, Lis C G, Grutsch J F. The Relationship Between Cancer-Related Fatigue and Patient Satisfaction with Quality of life in Cancer[J]. Journal of Pain \& Symptom Mangement. 2007;34(1):40-47.

3. Groenvold M, Petersen M A, Idler E, et al. Psychological distress and fatigue predicted recurrence and survival in primary beast cancer patients[J]. Breast Cancer Research \& Treatment. 2007;105(2):209219. 
4. Konkimalla VB, Efferth T. Evidence-based Chinese medicine for cancer therapy. J Ethnopharmacol. 2008;116(2):207-210.

5. Hofseth LJ, Wargovich MJ. Inflammation, cancer, and targets of ginseng. J Nutr. 2007;137(1 Suppl):183S-185S.

6. O'Higgins CM, Brady B, O'Connor B,Walsh D, Reilly RB. The pathophysiology of cancer-related fatigue: current controversies[J]. Supportive Care Cancer. 2018;26(10):3353-3364.

7. Wang XS. Pathophysiology of cancer-related fatigue. Clin J Oncol Nurs, 2008;12(5 Suppl): 11-20.

8. Cleeland CS, Bennett GJ, Dantzer R, Dougherty PM, Dunn AJ, Meyers CA, Miller AH, Payne R, Reuben $\mathrm{JM}$, Wang XS, Lee BN. Are the symptoms of cancer and cancer treatment due to a shared biologic mechanism? A cytokine-immunologic model of cancer symptoms. Cancer. 2003;97(11): 2919-2925.

9. Solomayer EF, Feuerer M, Bai L, Umansky V, Beckhove P, Meyberg GC, Bastert G, Schirrmacher V, Diel IJ. Influence of adjuvant hormone therapy and chemotherapy on the immune system analysed in the bone marrow of patients with breast cancer. Clin Cancer Res. 2003; 9(1):174-180.

10. Fitzgerald PB, Laird AR, Maller J, Daskalakis ZJ. A meta-analytic study of changes in brain activation in depression. Hum Brain Mapp, 2008; 29(6): 683-695.

11. Lamkin DM, Lutgendorf SK, Lubaroff D, Sood AK, Beltz TG, Johnson AK. Cancer induces inflammation and depressive-like behavior in the mouse: modulation by social housing. Brain Behav Immun. 2011; 25(3): 555-564.

12. Wang C, Feng L, Su J, Cui L, Dan Liu, Yan J, Ding C, Tan X, Jia X. Polysaccharides from Epimedium koreanum Nakai with immunomodulatory activity and inhibitory effect on tumor growth in LLCbearing mice. J Ethnopharmacol. 2017; 207:8-18.

13. Deng W, Zheng M, Zhang J, Huang C, Zhang Y. Immunologic functions of total flavone of Epimedium of two species in Guizho.Zhongguo Zhong Yao Za Zhi. 2011; 36(4): 511-513.

14. Etemadifar M, Sayahi F, Abtahi SH, Shemshaki H, Dorooshi GA, Goodarzi M, Akbari M, FereidanEsfahani M. Ginseng in the treatment of fatigue in multiple sclerosis: a randomized, placebocontrolled, double-blind pilot study. Int J Neurosci. 2013; 123(7): 480-486.

15. Mao XF, Piao XS, Lai CH, Li DF, Xing JJ, Shi BL. Effects of beta-glucan obtained from the Chinese herb Astragalus membranaceus and lipopolysaccharide challenge on performance, immunological, adrenal, and somatotropic responses of weanling pigs. J Anim Sci. 2005; 83(12):2775-2782.

16. Huang HL, Chen CC, Yeh $\mathrm{CY}$, Huang RL. Reactive oxygen species mediation of baizhu-induced apoptosis in human leukemia cells. J Ethnopharmacol. 2005; 97(1): 21-29.

17. Nikles S, Monschein M, Zou H, Liu Y, He X, Fan D, Lu A, Yu K, Isaac G, Bauer R. Metabolic profiling of the traditional Chinese medicine formulation Yu Ping Feng San for the identification of constituents relevant for effects on expression of TNF-a, IFN-ץ, IL $-1 \beta$ and IL-4 in U937 cells. J Pharm Biomed Anal. 2017; 145:219-229.

18. Zheng X. Clinical research guideline of new investigational drug in traditional Chinese medicine. Beijing: China Medical Science Press. 2002. 
19. International Conference on Harmonisation of technical requirements for registration of pharmaceuticals for human use. ICH harmonized tripartite guideline: Guideline for Good Clinical Practice. J Postgrad Med. 2001;47(1):45-50.

20. Sood A, Barton DL, Bauer BA, Loprinzi CL. A critical review of complementary therapies for cancerrelated fatigue. Integr Cancer Ther. 2007; 6(1): 8-13.

21. Terasawa K. Evidence-based reconstruction of Kampo medicine: part II-The concept of Sho. Evid Based Complement Alternat Med. 2004;1(2):119-123.

22. Cleeland CS, Bennett GJ, Dantzer R, Dougherty PM, Dunn AJ, Meyers CA, Miller AH, Payne R, Reuben $\mathrm{JM}$, Wang XS, Lee BN. Are the symptoms of cancer and cancer treatment due to a shared biologic mechanism? A cytokine-immunologic model of cancer symptoms. Cancer. 2003; 97(11): 29192925.

\section{Figures}

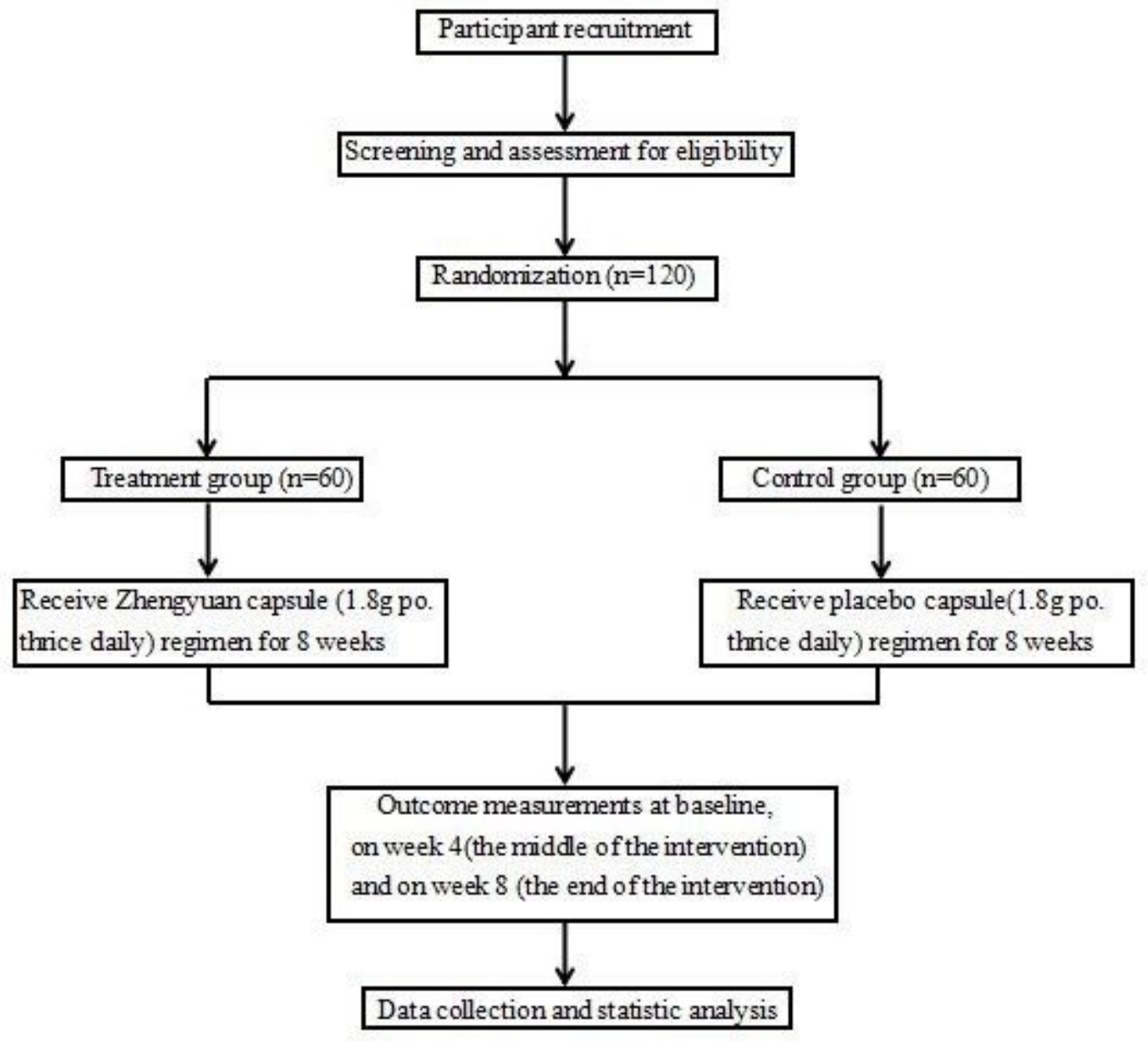

\section{Figure 1}

Study design flow chart. po. peros, Zhengyuan capsule 


\begin{tabular}{|c|c|c|c|c|}
\hline \multirow{3}{*}{ Research activities } & \multirow{2}{*}{$\begin{array}{l}\text { Screening } \\
\text { period }\end{array}$} & \multicolumn{3}{|c|}{ Treatment period } \\
\hline & & baseline & middle & end \\
\hline & $-7 \sim 0 \mathrm{D}$ & OD & $28 \pm 2 \mathrm{D}$ & $56 \pm 2 \mathrm{D}$ \\
\hline \multicolumn{5}{|c|}{ Basic information of collection and detemination of entry and discharge criteria } \\
\hline Sign the informed consent & $\boldsymbol{\Delta}$ & & & \\
\hline Review entry and discharge criteria & $\Delta$ & & & \\
\hline Demographic data & $\Delta$ & & & \\
\hline Physical examination & $\Delta$ & & & \\
\hline Medical history & $\Delta$ & & & \\
\hline Combined medication & $\Delta$ & $\boldsymbol{\Delta}$ & $\boldsymbol{\Delta}$ & $\boldsymbol{\Delta}$ \\
\hline CFS & $\boldsymbol{\Delta}$ & & & \\
\hline Pregnancy tests & & $\boldsymbol{\Delta}$ & & $\boldsymbol{\Delta}$ \\
\hline Random grouping & & $\Delta$ & & \\
\hline \multicolumn{5}{|c|}{ Effectiveness observation } \\
\hline CFS & & $\Delta$ & $\Delta$ & $\boldsymbol{\Delta}$ \\
\hline MFSI-SF & & $\Delta$ & $\Delta$ & $\boldsymbol{\Delta}$ \\
\hline TCM syndrome pattem differentiation & & $\boldsymbol{\Delta}$ & $\boldsymbol{\Delta}$ & $\boldsymbol{\Delta}$ \\
\hline Levels of immumologic indicators & & $\Delta$ & & $\Delta$ \\
\hline Pulmonary function & & $\Delta$ & $\Delta$ & $\boldsymbol{\Delta}$ \\
\hline \multicolumn{5}{|c|}{ Safety observation } \\
\hline Vital signs & $\boldsymbol{\Delta}$ & $\Delta$ & $\Delta$ & $\Delta$ \\
\hline Blood routine & & $\Delta$ & & $\boldsymbol{\Delta}$ \\
\hline Urine routine and urinary sediment & & $\Delta$ & & $\boldsymbol{\Delta}$ \\
\hline Liver function and Kidney function & & $\Delta$ & & $\Delta$ \\
\hline Electrocardiogram & & $\boldsymbol{\Delta}$ & & $\boldsymbol{\Delta}$ \\
\hline \multicolumn{5}{|c|}{ Other jobs } \\
\hline Distribute test drugs & & $\Delta$ & $\Delta$ & \\
\hline Drug recovery and inventory & & & $\Delta$ & $\Delta$ \\
\hline Compliance records & & & $\Delta$ & $\Delta$ \\
\hline Make an appointment for a follow-up visit & & $\Delta$ & $\Delta$ & \\
\hline Adverse events & $\boldsymbol{\Delta}$ & $\Delta$ & $\Delta$ & $\boldsymbol{\Delta}$ \\
\hline
\end{tabular}

Figure 2

Treatment schedule and outcome measures. D day, Zhengyuan capsule

\section{Supplementary Files}

This is a list of supplementary files associated with this preprint. Click to download.

- renamedeaa06.doc 